Service social

\title{
Intervention de groupe auprès des conjoints violents et logique du contrôle social
}

\section{Pierre Turcotte}

Volume 46, numéro 2-3, 1997

Groupes - Symposium 1997

URI : https://id.erudit.org/iderudit/706768ar

DOI : https://doi.org/10.7202/706768ar

Aller au sommaire du numéro

Éditeur(s)

École de service social de l'Université Laval

ISSN

1708-1734 (numérique)

Découvrir la revue

Citer cet article

Turcotte, P. (1997). Intervention de groupe auprès des conjoints violents et logique du contrôle social. Service social, 46(2-3), 227-239.

https://doi.org/10.7202/706768ar d'utilisation que vous pouvez consulter en ligne.

https://apropos.erudit.org/fr/usagers/politique-dutilisation/ 


\section{Intervention de groupe auprès des conjoints violents et logique du contrôle social}

Pierre TURCOTTE

La recherche sur l'intervention auprès des conjoints violents est abondante en ce qui concerne l'efficacité et l'impact des programmes de thérapie (Lindsay, Ouellet et Saint-Jacques, 1993; Rondeau, 1994), la catégorisation des agresseurs et de leur personnalité (Gondolf, 1988), les différentes approches utilisées ainsi que les cadres d'analyse qui les supportent (Burns et Meredith, 1991; Lindsay et Ouellet, 1992). Bref, de nombreuses études se sont intéressées à la pertinence et aux résultats de l'intervention (le pourquoi et le comment) en violence conjugale. Toutefois, les travaux sur l'analyse de la violence conjugale en tant que problème social sont plus limités.

Basé sur l'analyse du discours des intervenants de deux organismes communautaires de Montréal œuvrant dans le domaine de la violence conjugale (OPTION et PRO-GAM), ce texte tente de démontrer que l'aide aux conjoints violents s'inscrit dans une logique de contrôle social. Dans un premier temps, la violence conjugale est située comme un problème social, à savoir : une construction sociale fondée sur un discours idéologique s'appuyant sur des valeurs d'égalité entre les sexes et de défense des droits civiques. La criminalisation a été la réponse sociale à ce phénomène dénoncé et rendu visible par le mouvement d'émancipation des femmes, participant ainsi à la constitution d'un nouveau problème social : la violence conjugale. Par la suite, la portée de la judiciarisation de la violence conjugale sur l'intervention sociale est discutée pour 
déboucher sur quelques réflexions sur les liens entre l'intervention thérapeutique et le contrôle social.

\section{LA VIOLENCE CONJUGALE COMME PROBLÈME SOCIAL}

Faire d'un phénomène humain particulier un problème social, c'est déjà le situer dans une dynamique non seulement de dysfonctionnement, mais également de création de sens. On donne alors à ce phénomène un surplus de sens, puisqu'il devient un problème particulier que la société doit résoudre. La constitution d'un problème social passe par un travail d'identification des acteurs, de leurs rôles et de leurs interactions. Des discours de légitimation viennent s'y ajouter, discours ayant un double effet : un effet de dramatisation de l'objet phénoménologique et un effet de constitution d'un pouvoir d'expertise tant sur la définition que sur la résolution du problème.

Selon la définition donnée par Lenoir (1989), un problème social est une réalité préconstruite socialement. II reflète un phénomène qui préexiste à sa constitution en tant que problème socialement reconnu. En fait, les phénomènes qu'une société constitue en " problèmes sociaux " varient selon les époques et les régions. Ces problèmes sociaux peuvent même disparaître, alors même que les phénomènes qu'ils désignent, eux, subsistent. II en est ainsi, par exemple, de la pauvreté, qui fut aux États-Unis un grave problème social pendant les années 1930 et disparut dans la décennie 19401950 , ou encore du racisme qui ne deviendra un problème social que dans les années 1960 (Lenoir, 1989).

De plus, la réaction d'une société à un problème social varie à la fois en fonction du champ d'action où il est défini (politique, économique, psychosociologique ou culturel) et de la nature des réponses qui sont apportées. Ces réponses peuvent être financières (compensation des accidents du travail, etc.), prendre la forme d'assurances (sécurité du revenu, assurance-maladie, etc.), donner lieu à des gestes de solidarité (réseaux d'entraide, services humanitaires, etc.) ou s'appuyer sur le contrôle social.

Au début du siècle, le contrôle social était associé à la capacité d'une société à se réguler elle-même (Robert, 1982). Par la suite, ce concept a évolué, se référant à des " appareils [...] ou, plus largement [...] des lieux et des pratiques généralement contrôlés par l'État 
et impliquant une domination idéologique et répressive ayant pour but le développement d'une société disciplinaire normalisante " (Robert, 1982).

\section{LA JUDICIARISATION DE LA VIOLENCE CONJUGALE}

S'appuyant entre autres sur la Charte des droits et libertés, le mouvement d'émancipation des femmes a réclamé la criminalisation des actes d'agression intra-familiale, au même titre que les agressions entre adultes étrangers (CCCSF, 1991). La lutte pour obtenir l'application du Code criminel sans distinction quant aux liens entre agresseurs et victimes a mis en branle le traitement judiciaire des cas d'agression entre conjoints. Ce faisant, le mouvement féministe a contribué à amener la réponse sociale à la violence conjugale dans la voie de ce que Horwitz (1990) appelle le contrôle social pénal.

Les enjeux politiques, institutionnels et sociaux qui entourent la criminalisation et la judiciarisation de la violence conjugale doivent être saisis d'abord autour de la définition de ce phénomène. Spontanément, on pourrait penser que violence conjugale signifie "les agressions d'un conjoint contre l'autre au sein du couple ". Toutefois, à la manière dont les acteurs ont défini l'objet, on constate qu'il ne s'agit pas seulement d'agressions, que l'agresseur n'est pas n'importe quel conjoint et que cette violence ne se situe pas dans n'importe quel couple. En 1987, les documents ministériels circonscrivaient la violence conjugale à la seule violence des hommes envers leur conjointe (Québec, 1987) : pour les auteurs du document, la violence conjugale, c'est de la violence masculine hétérosexuelle.

Par ailleurs, la reconnaissance de la violence conjugale comme un crime, ainsi que l'énonce le document ministériel Orientations (Québec, 1992), devrait limiter la définition de la violence aux seuls actes constituant des infractions définies par le Code criminel canadien. Or, la violence conjugale est vue, dans ce même document ministériel, de façon à inclure tous les comportements conflictuels entre conjoints qui sont considérés comme répréhensibles : « Toutes les formes d'agression (verbale, psychologique, physique, sexuelle, socio-économique...) doivent être condamnées socialement. Le fait que la violence entraîne ou pas des poursuites judiciaires ne doit pas en changer fondamentalement le caractère répréhensible, ni le traitement " (Québec, 1992). 
Cette prise de position traduit le caractère symbolique de la criminalisation de la violence conjugale. En effet, en criminalisant les agressions entre adultes au sein d'une relation intime, la société utilise la forme de contrôle social la plus répressive et la plus claire symboliquement. II s'agit de la constitution d'une action en une infraction, ce que Acosta (1987) a appelé la mise en forme pénale d'un événement social.

\section{LA PORTÉE DE LA JUDICIARISATION SUR L'INTERVENTION SOCIALE}

La mise en application de cette criminalisation se concrétise à travers deux principaux systèmes : le social et le judiciaire. La concomitance de ces deux systèmes conduisant à la judiciarisation pour un et à l'intervention sociale pour l'autre crée ce que l'on a appelé au Québec la sociojudiciarisation (Morier et collab., 1981) des agresseurs en violence conjugale.

Au Québec, les premiers services d'aide psychosociale pour conjoints violents au Québec sont issus, pour bon nombre d'entre eux, des groupes de condition masculine du début des années 1980, regroupés autour de collectifs comme Hommes-Info, le Collectif masculin contre la violence domestique et Cœur-Atout (Rondeau, 1989). Les premiers groupes de thérapie au Québec sont mis en place à Montréal vers 1982, puis à Laval (CHOC) et Sherbrooke (Le Seuil de l'Estrie) en 1985. En 1988, ils se regroupent autour d'un organisme provincial - l'ARIHV (Association des ressources intervenant auprès des hommes violents) - qui adoptera l'analyse féministe selon laquelle la violence conjugale trouve son origine dans l'inégalité des pouvoirs entre les hommes et les femmes dans une société patriarcale et sexiste. Cette analyse servira d'ailleurs de base aux représentants de l'ARIHV lors de leur participation au comité consultatif ministériel ayant pour mandat de rédiger une politique ministérielle sur l'intervention auprès des conjoints violents, politique qui sera rendue publique en février 1992 (Intervention auprès des conjoints violents : Orientations).

Sur le plan clinique, toutefois, les choses sont beaucoup plus complexes. Il existe au Québec un certain pluralisme sur le plan des approches d'intervention : approches systémique, cognitivo-comportementale, psycho-dynamique et proféministe (Rondeau et collab., 1989). Plusieurs organismes contestent aujourd'hui ces appellations 
trop limitatives; la plupart disent plutôt s'inspirer de plusieurs modèles et de différentes approches thérapeutiques. Cet éclectisme tient compte, d'une part, de la complexité de la problématique et de l'évolution de la connaissance développée par l'intervention et, d'autre part, de l'analyse sociale que ces intervenants font de la violence conjugale. Leur action a cependant un point commun : elle privilégie l'intervention de groupe. En effet, l'intervention individuelle est jugée inefficace pour des clients qui ne se considèrent pas comme ayant un problème de violence et qui réagissent par le déni, la culpabilisation de la conjointe, etc.

II y a deux types de participants aux groupes de thérapie : les clients volontaires, qui s'inscrivent à la thérapie de leur propre chef, bien que cette démarche résulte généralement de plusieurs facteurs comme les pressions ou les menaces de la conjointe ou de l'entourage, et les judiciarisés, qui ont été interpellés par la police et mis en accusation à la suite d'un acte de violence conjugale (Fournier, 1992).

Cette catégorisation ne rend toutefois pas vraiment compte de la réalité, car les volontaires, bien que n'étant dirigés vers une thérapie par aucun système extérieur, y participent souvent à la demande expresse de leur conjointe ou de leur environnement familial. Par contre, et ce qui reflète bien ce que Horwitz pose comme lieu volontaire du contrôle social thérapeutique, " le libre consentement et la participation volontaire de personnes dysfonctionnelles » (Horwitz, 1990, p. 80) ${ }^{1}$, tous ces clients doivent collaborer au processus et être d'accord pour entreprendre un processus thérapeutique en signant une entente avec le thérapeute. C'est ce que les organismes appellent le contrat thérapeutique.

L'existence de ces groupes d'aide pour conjoints violents, bien qu'antérieure à la criminalisation de la violence conjugale, fut dès le début liée d'une certaine façon à la réponse sociale, qui est une réponse de contrôle social pénal. En effet, les premiers intervenants auprès des conjoints violents furent étroitement associés à la rédaction de la Politique d'intervention en matière de violence conjugale publiée en 1986 par le ministère de la Justice et le ministère du Solliciteur général; cette politique élabore le modèle de l'intervention

1. Traduction de l'auteur. 
sociojudiciaire, introduisant la complémentarité du traitement judiciaire et des programmes de thérapie.

Cette conception normative de contrôle social correspond à la vision du sociologue américain Allan Horwitz (1990), à savoir une conception qui rend compte de cette partie de la socialité où se mettent en œuvre les actions de conformité aux normes socialement construites du comportement humain. Si la socialisation est le lieu de l'acquisition par l'individu des normes de comportement, le contrôle social est celui où s'opère la gestion de la déviance à ces normes. Horwitz énonce que le contrôle social peut s'opérer selon plusieurs modes. II en développe quatre : les modes pénal, médiatoire, compensatoire et thérapeutique. Je n'en utiliserai ici que deux : le mode pénal et le mode thérapeutique. Par contrôle social pénal, Horwitz entend le processus par lequel la société en arrive à juger et à punir les agresseurs qui ont contrevenu à des règles comportementales condamnées et légalement codifiées sous forme d'infractions. Le mode pénal du contrôle social se situe ainsi au niveau moral et il se concrétise par la judiciarisation de comportements considérés comme des infractions au Code criminel.

Le contrôle pénal est un mode se situant au niveau de la morale et vise à punir les contrevenants qui ont outrepassé certaines normes légales ou extra-légales de comportement. L'aspect fondamental du mode pénal est d'infliger une souffrance ou d'imposer toute autre conséquence négative aux contrevenants qui ont commis des actes répréhensibles... Parce que les sanctions pénales ont pour but de punir les contrevenants, le mode pénal de contrôle social est inévitablement coercitif, plutôt que persuasif (Horwitz, 1990, p. 23-24)2.

Horwitz situe par ailleurs le mode thérapeutique de contrôle social au niveau de la modification des comportements socialement prohibés dans un contexte de coopération thérapeute/client plutôt que celui d'une obligation légale :

Le contrôle social thérapeutique modifie la personnalité de personnes dysfonctionnelles... Les intervenants du contrôle social thérapeutique s'efforcent de ramener leurs patients à la normalité par la manipulation de systèmes symboliques. Les patients doivent coopérer avec les thérapeutes pour que le processus de

2. Traduction de l'auteur. 
guérison puisse réussir. Le résultat attendu n'est pas une directive donnée à une partie adverse, ni le respect d'une obligation ou la réparation des dommages causés à quelqu'un, mais le changement d'une personnalité dysfonctionnelle (Horwitz, 1990, p. 79$)^{3}$.

J'émets l'hypothèse que le mode de contrôle social présent dans l'intervention de groupe auprès des conjoints violents est plutôt de type thérapeutique. II s'agit néanmoins d'un mode de contrôle social qui est, selon les cas, soit concomitant (le client vient volontairement à la thérapie), soit en prolongation du mode pénal (« référence » par la Cour à la suite d'une sentence).

Un des problèmes qui préoccupent les organismes intervenant auprès des conjoints violents concerne le moment où l'on doit admettre un client judiciarisé en groupe de thérapie. L'un de ces organismes, OPTION, admet les clients judiciarisés à n'importe quel moment du processus judiciaire. Toutefois, il se réserve le droit de retourner au système judiciaire un client « référé » qui ne collabore pas suffisamment à sa thérapie : on parle de stratégie d'intervention intersystémique. D'autres organismes, tel PRO-GAM ${ }^{4}$, préfèrent attendre la fin du processus judiciaire avant d'admettre un client en groupe de thérapie. L'une des raisons invoquées est la motivation biaisée du client à l'égard de la thérapie et son utilisation des services de l'organisme à des fins de défense judiciaire.

L'analyse du discours des intervenants d'OPTION permet d'associer leur système thérapeutique au mode pénal de contrôle social. Quand il est question d'aide ou de thérapie, ils veulent s'assurer que cela n'ouvre pas la voie à une quelconque forme de décriminalisation. Par l'importance des liens mutuels de renvoi entre les systèmes thérapeutique et judiciaire, cette position vient théoriser et justifier la complémentarité des deux modes pénal et thérapeutique de contrôle social. En ce sens, elle reflète l'approche sociojudiciaire de l'intervention auprès des conjoints violents.

3. Traduction de l'auteur.

4. Depuis quelque temps, PRO-GAM offre des services pour la clientèle judiciarisée à travers des groupes de thérapie spécialisés regroupant uniquement cette clientèle. 
D'un autre côté, le discours des intervenants de PRO-GAM reflète davantage l'utilisation d'un mode psychothérapeutique ${ }^{5}$ du contrôle social. Ce faisant, il se situe en marge du discours dominant sur la violence conjugale où le contrôle pénal est privilégié. En effet, les intervenants de PRO-GAM insistent sur la primauté de l'aide psychosociale sur le contrôle pénal. Leur discours, qui accorde une légitimité au mode thérapeutique de contrôle social, autorise la prise en compte du vécu des agresseurs. Bien qu'ils soient totalement responsables de leurs actes de violence, les contrevenants sont vus comme des personnes ayant besoin d'aide, comme des acteurs conscients qui ont fait le choix d'utiliser la violence, mais qui peuvent faire d'autres choix grâce à l'accompagnement adéquat que constitue la thérapie.

Comment comprendre que des hommes qui tiennent à leur conjointe (parfois même pathologiquement, par dépendance affective) et à leurs enfants en soient rendus à les violenter? II y a manifestement une dimension psychosociale dans le comportement de ces hommes. En cela, ils ne diffèrent pas, comme type de criminels, de ceux et celles qui maltraitent ou abusent de leurs enfants, en ce sens qu'eux aussi ils ont un rapport intime avec la victime.

Dans leur discours, les intervenants de PRO-GAM insistent sur l'importance "de passer par l'expérience individuelle, personnelle et intime pour [...] rejoindre l'individu [...] qu'il se sente reçu [...] et qu'il accepte de s'ouvrir à son expérience ". La trajectoire du conjoint violent participant à une thérapie s'inscrit dans la logique du contrôle social thérapeutique, mais la thérapie, qui se fait dans un climat d'accueil, d'empathie et de respect, lui permet de se voir reconnu comme être humain d'abord. Ainsi, quand le conjoint violent décide de consolider lui-même sa décision de changement, une logique autre que le changement comportemental et qui est de l'ordre de l'attitude et des valeurs peut s'installer. Cette intervention se fonde

5. J'utilise cette expression pour mieux démarquer le mode plus psychosocial que médical de contrôle social en présence dans les interventions de l'organisme étudié. En effet, à la lecture de la définition de Horwitz, on a tendance à y percevoir une conception psychiatrique de l'aide supposant que le client souffre d'une maladie. Le modèle psychosocial d'aide considère le client comme un adulte responsable apte à faire des choix, dont son propre changement. 
sur le pouvoir personnel de changement des clients eux-mêmes et non dans la seule conformité au discours social en matière de prescription de comportements.

Bien que ces deux organismes privilégient un mode de contrôle différent, l'analyse de leurs discours respectifs démontre que leur intervention s'inscrit tout à fait dans la logique du contrôle social. Cette logique est inhérente à la constitution même de la réponse sociale à la violence conjugale, à savoir la protection des victimes et la répression des agresseurs.

On ne peut pas échapper à cette logique, à moins de déconstruire complètement la problématisation actuelle du phénomène des agressions entre conjoints telle que décrite par la politique gouvernementale en matière de violence conjugale (Québec, 1995). Toutefois, tenter de comprendre le sens que prend le contrôle social dans le discours sur la violence conjugale et discerner les modes pénal et thérapeutique comme étant distincts mais concomitants dans leur application concrète, c'est déjà participer au processus de reconstruction de ce problème social en mettant l'accent sur le type de réponse à y apporter.

\section{INTERVENTION THÉRAPEUTIQUE ET CONTRÔLE SOCIAL}

Les deux positions mises en évidence dans ces discours laissent voir la concomitance de deux modes de contrôle social (thérapeutique et pénal), témoignant en cela, d'une part, de la complexité du phénomène et, d'autre part, des efforts des acteurs pour trouver des solutions appropriées à ce problème. L'utilisation de l'un ou l'autre de ces deux modes du contrôle social reflète en partie les grands enjeux politiques liés à la problématisation de la violence conjugale, à savoir choisir entre traiter des individus ayant des comportements violents à l'égard de leur conjointe ou punir des agresseurs qui s'arrogent le droit d'utiliser la force et la contrainte pour contrôler leur partenaire.

Le concept de contrôle social, comme lieu de gestion de la conformité aux normes construites socialement, subsume la régulation sociale, voire s'y substitue, mais n'épuise pas tout le champ de la socialisation. La logique du contrôle social a ses limites : elle ne permet que la conformité à des normes comportementales socialement 
construites. Mais, comme on l'a vu dans l'analyse des discours des intervenants des deux organismes étudiés, il n'y a pas que le contrôle social. L'attitude empathique des intervenants de PRO-GAM et leur souci de créer une alliance thérapeutique avec leurs clients, de même que l'action du système-intervention d'OPTION qui vise à faire se réapproprier la demande de thérapie par les clients, illustrent que, dans la pratique même de l'intervention et dans les interstices de la relation client-intervenant, il y a place pour le changement social.

Comme le souligne Dulac (1997a), la socialisation des hommes est un facteur puissant d'inhibition de la demande d'aide. Or, lorsqu'un homme s'inscrit dans un processus thérapeutique, il transgresse une norme de la masculinité puisqu'il reconnaît son incapacité à résoudre seul son problème. Dès ce moment, il émerge chez ces hommes de nouvelles normes comportementales et identitaires. Le processus de changement mis en branle n'est pas seulement individuel, mais social et culturel.

\section{CONCLUSION}

La criminalisation de la violence conjugale a eu pour conséquence imprévue la création d'un large réseau de ressources s'adressant spécifiquement aux hommes. Bien que ce réseau offre des services pour des hommes ayant des comportements dysfonctionnels, il rend disponible une réponse qui interpelle les clients comme déviants.

Malgré la préséance dans le discours de l'État (Québec, 1995) du contrôle social sur toute autre forme de réponse aux problèmes sociaux, illustrant ainsi ce que Duchastel et Laberge (1990) ont appelé les transformations étatiques des modes de contrôle social, l'intervention sociale auprès des conjoints violents permet et autorise chez les intervenants un espace d'autonomie relativement à la perception du client vu comme acteur responsable, faisant des choix, donc ayant le pouvoir de choisir le changement. De déviants aux normes sociales, les clients deviennent alors déviants à la norme identitaire masculine qui condamne la demande d'aide : plus que modification d'un comportement, il y a possibilité pour le client acteur de créer de nouvelles façons de se vivre au masculin.

Pour sortir de la logique où les seuls services offerts aux hommes sont des services axés sur des problèmes dysfonctionnels, il 
nous semble qu'il faille aller en amont de l'apparition des comportements, à savoir au niveau de la constitution et de la promotion de nouvelles normes identitaires masculines, comme le proposent le Réseau Hommes Québec ainsi que l'intervention dans le domaine de la paternité (Dulac, 1997b). Ce faisant, on pourra accorder aux hommes le droit de dévier des normes masculines traditionnelles véhiculant l'autonomie à tout prix et la non-expression des émotions négatives comme la peine, la peur, la douleur.

Je voudrais terminer en rapportant cette phrase prophétique et remplie d'espoir de Tennessee Williams, cité dans le dépliant promotionnel de GAPI ${ }^{6}$ :

J'ai l'impression d'avoir vécu toute ma vie les poings bien fermés : pour cogner dur, massacrer et foncer.

À partir de maintenant, je veux desserrer les poings, ouvrir les mains et toucher tout doucement.

Pierre TURCOTTE

Travailleur social

Organisme Action sur la violence et intervention familiale (AVIF)

Châteauguay, Québec

6. Groupe d'aide aux personnes impulsives (ayant des comportements violents), organisme communautaire d'aide aux conjoints violents de la région de Québec. 


\section{Références bibliographiques}

AcostA, F. (1987). « De l'événement à l'infraction : le processus de mise en forme pénale ", Déviance et Société, vol. 11, n 1, p. 1-40.

Burns, N., C. MEREdiTH et C. PAQUetTe (1991). Programmes de traitement pour les hommes violents : une analyse qui témoigne de leur succès, Ottawa, ministère de la Justice du Canada.

CASTEL, R. (1988). « De l'intégration sociale à l'éclatement du social : l'émergence, l'apogée et le départ à la retraite du contrôle social ", Revue internationale d'action communautaire, 20/60, p. 67-77.

CONSEIL CONSULTATIF CANADIEN SUR LA SITUATION DE LA FEMME (1991). La violence faite aux femmes par les hommes : la brutalité de l'inégalité, Ottawa, CCCSF.

DUCHASTEL, J. et D. LABERGE (1990). “ Transformation des modes étatiques de contrôle social », Politique, 20, p. 65-92.

DULAC, G. (1997a). Les demandes d'aide des hommes, Montréal, Université McGill, AIDRAH.

DULAC, G. (1997b). « La configuration du champ de la paternité : politiques, acteurs et enjeux ", Lien social et Politiques, 37, printemps 1997, Montréal, RIAC, p. 133-143.

FOURNIER, A. (1992). Engagement des hommes dans un traitement contre la violence conjugale : recherche de différences entre volontaires et judiciarisés. Mémoire de maîtrise, Montréal, Université du Québec à Montréal.

Horwitz, A. (1990). The Logic of Social Control, New York, Plenum Press.

LENOIR, R. (1989). "Objet sociologique et problème social ", dans P. Champagne, Initiation à la pratique sociologique (p. 54-99), Paris, Dunod.

LINDSAY, J. et F. OUELLET (1992). Recension des écrits concernant l'évaluation des programmes de traitement pour conjoints violents, Québec, CRSC.

LINDSAY, J., F. OUELLET et M.-C. SAINT-JACQUES (1993). Évaluation de l'efficacité d'un programme de traitement pour conjoints violents, Québec, CRSC.

MORIER, Y. et collab. (1991). Intervention sociojudiciaire en violence conjugale, Montréal, Wilson et Lafleur. 
QuÉbec. MINISTÈre de LA SANTÉ ET DES SerVICES SOCIAUX, MINISTÈre DE LA JUSTICE, SECRÉTARIAT À LA CONDITION FÉMININE, MINISTĖRE DE LA SÉCURITÉ PUBLIQUE, MINISTĖRE DE L'ÉDUCATION Et SECRÉTARIAT À LA FAMILLE (1995). Politique d'intervention en matière de violence conjugale, prévenir, dépister, contrer la violence conjugale, Québec, Les Publications du Québec.

QUÉBEC. Ministère dE LA SANTÉ ET DES SERVICES SOCIAUX (1992). Intervention auprès des conjoints violents : Orientations, Québec, Les Publications du Québec.

QuÉBEC. Ministère de LA SANTÉ eT DES SERVICES SOCIAUX (1987). Une politique d'aide aux femmes violentées (éd. révisée), Québec, Les Publications du Québec.

QUÉBEC. MINISTÈRE DE LA JUSTICE ET MINISTÈRE DU SOLLICITEUR GÉNÉRAL DU QUÉBEC (1986). Politique d'intervention en matière de violence conjugale, Québec, Les Publications du Québec.

ROBERT, P. (1982). " De la "criminologie de la réaction sociale" à une sociologie pénale ", L'Année sociologique, 31, p. 253-283.

RONDEAU, G. et collab. (1989). Les programmes québécois d'aide aux conjoints violents : rapport sur les 16 organismes existant au Québec, Ministère de la Santé et des Services sociaux, Direction générale de la planification.

RONDEAU, G. (1994). « La violence familiale ", dans F. Dumont, S. Langlois et Y. Martin (dir.), Traité des problèmes sociaux (p. 319-335), Québec, IQRC.

TURCOTTE, P. (1996). Intervention auprès des conjoints violents judiciarisés : une forme de contrôle social de type pénal ou thérapeutique. Analyse des discours de deux organismes communautaires. Mémoire de mâ̂trise en sociologie, Montréal, UQAM, $107 \mathrm{p}$. 